\title{
Conception and Clinical Efficacy of an Osmotic, Polymeric, Stable Nasal Filmogen Barrier for Preventive Treatment of Allergen and Pollution Induced Rhinitis and Respiratory Symptoms
}

\author{
Rémi Shrivastava $^{1}$ (Ph.D.), Nathalie Maneby ${ }^{1}$, Gilles Giroir ${ }^{1}$, Marjorie Georges ${ }^{1}$, Amit Bhatt $^{2}$ and Ravi Shrivastava $^{{ }^{*}}$ \\ ${ }^{1}$ Vitro-Bio Research Institute, Issoire, France. \\ ${ }^{2}$ Nexus Clinical Research LLC, 08 Valley Road Ithaca, NY 14850 - USA.
}

*Corresponding Author: Ravi Shrivastava, Vitro-Bio Research Institute, Issoire, France.

Received date: December 01, 2021; Accepted date: December 17, 2021; Published date: January 05, 2022

Citation: Ravi Shrivastava, Rémi Shrivastava, Nathalie Maneby, Gilles Giroir, Marjorie Georges, et al. (2022). Conception and Clinical Efficacy of an Osmotic, Polymeric, Stable Nasal Filmogen Barrier for Preventive Treatment of Allergen and Pollution Induced Rhinitis and Respiratory Symptoms. J Clinical Research and Reports, 10(1); DOI:10.31579/2690-1919/219

Copyright: (C) 2022, Ravi Shrivastava. This is an open access article distributed under the Creative Commons Attribution License, which permits unrestricted use, distribution, and reproduction in any medium, provided the original work is properly cited.

\begin{abstract}
Background: Pollution induced allergic rhinitis and respiratory symptoms is becoming a major health problem in the world for which still there is no safe and preventive treatment.

Objectives: Conceive and evaluate the allergen preventive properties and clinical efficacy of an osmotic, polymeric, stable filmogen spray, called PCNS.

Materials and Methods: Amb A 1 (ragweed), Der P 1 and 2 (dust mite), Bet v 1 (birch), Alt a 1 (Alternaria, fungus), and Fel d 1 (cat dander) allergens were exposed at a concentration of $5 \mu \mathrm{g} / \mathrm{ml}(20 \mu \mathrm{l}$ per tube) on the polymeric test product film (120 and 240 $\mu$ l layer) and the allergens crossing the barrier were quantified in the agar gel beneath the film. 0.40\% HPMC and PBS solutions, tested identically, served as controls. Clinical efficacy of PCNS nasal spray was evaluated in patients suffering from allergic rhinitis and/or respiratory symptoms (29 in test product v/s 15 in saline controls) for 22 days. Nasal, ocular, respiratory symptoms and Rhino conjunctivitis Quality of Life Questionnaire (RQLQ) were measured. Statistical analyses: The normality of the populations was determined by the Shapiro-Wilk test, then statistical analysis was performed by two-tailed Student's test for comparisons between two groups and the two-way ANOVA followed by the post hoc Bonferroni's test for comparisons of multiple groups. p $<0.05$ was considered statistically significant. The analyses were performed with the software GraphPad Prism (version 8.4.2, La Jolla, USA). NS indicates not significant.
\end{abstract}

Results: PCNS polymeric spray blocked the diffusion of all the allergens while $0.40 \%$ HPMC was able to prevent diffusion of only Alt a 1 and Fel d 1 allergens. Mean reflective total nasal symptom scores (rTNSS), reflective total ocular symptom score (rTOSS), and respiratory symptoms including effect on wheezing, cough, dyspnea, and chest tightness were moderately improved in the control saline group, but the improvements were nearly twice better in the PCNS group. RQLQ was improved by $23 \%$ in saline spray v/s 46\% PCNS group. 4/15 patients in saline group v/s $1 / 29$ in PCNS group required rescue medication during the study. PCNS was highly effective in reducing allergen and pollution induced respiratory symptoms.

Conclusion: a polymeric, osmotic, and stable nasal barrier against pollutants and allergens represents an innovative approach against pollution induced respiratory symptoms.

Short title / running head: Nasal barrier film against allergic rhinitis and respiratory symptoms

Keywords: nasal barrier; polymeric; allergen; pollution; respiratory symptoms 


\section{Introduction}

A large number of epidemiological studies have demonstrated a dramatic increase in the prevalence of allergic diseases over the last 10-20 years throughout the world with prevalence up to $50 \%$ in some countries $[3,8]$. Common allergic rhinitis (AR) symptoms are characterized by sneezing, nasal congestion, nasal itching and rhinorrhoea (nasal discharge) which may be followed by respiratory symptoms in chronic cases. Most allergenic particles are present in the environment and usually encounter human body while respiration. The nasal mucosa (NM) is generally the primary site of contact with allergens and also the first line of defense [9].

The most common allergens include pollens, minute vegetable proteins in the environment, weed, house dust, mites, molds, and animal-related allergens [34]. Due to the excessive vehicle exhaust fumes and industrial activity, about 55\% world population living in congested cities is continuously exposed to polluted urban air containing diesel exhaust particles (DEPs), high-molecular weight organic chemical components and heavy metals such as $\mathrm{As}, \mathrm{Pd}, \mathrm{Cd}, \& \mathrm{Hg}$ which become potential allergens [16]. These toxic particles damage airway mucous membranes and impair mucociliary clearance, facilitating presentation of inhaled allergens to the immune cells, thus promoting sensitization of the airway [17]. Consequently, a more severe immunoglobulin (Ig) E-mediated response to aeroallergens and airway inflammation could account for increasing prevalence of allergic respiratory diseases in polluted urban areas. AR, asthma and atopic dermatitis often coexist in the same individual, partially due to a shared genetic origin [2, 14].

In the presence of conditions or factors that impair NM integrity, the allergens are recognized by dendritic cells. These cells trigger a series of events leading to the generation of plasma cells that produce allergenspecific immunoglobulin E ( $\operatorname{IgE}$ ) which binds to mast cells \& basophils and to a pool of memory allergen-specific type $2 \mathrm{~T}$ helper cells ( $\mathrm{T}_{\mathrm{H}} 2$ cells) and $\mathrm{IgE}^{+} \mathrm{B}$ cells. In individuals who are sensitized to the allergen, subsequent allergen exposures directly activate basophils and mast cells in the NM, triggering the release of allergic mediators such as histamine, leukotrienes, and proinflammatory cytokines. This nearly instant cytokine production by memory allergen-specific $\mathrm{T}_{\mathrm{H}} 2$ cells induces NM inflammation, membrane damage, pain, itching, irritation and chronic rhinitis [10,22]. NM plays a key role in this complex disease physiopathology which may further progress to asthma and other respiratory complications (Watts et al. 2019). Therefore, maintaining and restoring NM integrity to minimize allergen induced complex respiratory pathology may constitute the most logical approach for the prevention of AR.

Allergens with protease activity (dust mites -Der p 1 and 2) are highly cytotoxic to the epithelial barrier, whereas other common allergens can activate pattern recognition receptors and instant trigger of immune inflammation. Other allergens such as Amb A 1 (ragweed), Bet v 1 (birch-), Alt a 1 (Alternaria, fungus allergens), Fel d 1 (cat dander), Phl p 5 (timothy grass) and Blot $\mathrm{t} 5$ (storage mite), act identically by initiating immune response and inflammation through the release of cytokines such as IL-33, thymic stromal lymphopoietin (TSLP) and IL-25 ) [22]. These cytokines, in turn, activate group 2 innate lymphoid cells (ILC2s), release other proteins (IL-5, IL-13 and IL-4), and favor generation of specific IgE antibodies. IgE molecules bind to mast cells and trigger the release of histamine, heparin and leukotrienes (Shamji et al. n.d.).

When symptoms appear, the individual is already sensitized, and the disease has already become multi-factorial and chronic. AR lasts up to the time the allergens are present in the environment. Therefore, treating such a chronic, complex, and multi-factorial disease with repeated administration of chemical or biological drugs cannot be safe, but almost all the currently used treatments belong to this category. The commonly used $\mathrm{H}_{1}$-antihistamines, through oral, intranasal, or ocular formulations block the action of histamine by acting as neutral receptor antagonists while intranasal corticosteroids (INCS) such as beclomethasone, budesonide, ciclesonide, fluticasone propionate, and others reduce inflammation by constructing NM blood vessels and minimizing the influx of inflammatory mediators in response to allergic stimuli. An association of both these drugs is usually prescribed to treat severe cases of AR [4].

Leukotriene receptor antagonists, montelukast and zafirlukast, are also used in the treatment of AR and their effect is close to that of oral $\mathrm{H}_{1}$ antihistamines. Cetirizine, a non-drowsy antihistaminic is commonly used in children. New biological drugs against asthma and allergy can block only one protein and can be used only in specific severe cases of AR [13].

Such multi-factorial upper respiratory tract (URT) diseases are difficult to treat as it requires stopping allergen contact or using a multi-target approach which should not only protect the NM against new allergen contact but also block histamine, neutralize pro-inflammatory cytokines, and clean the NM all types of contaminants to prepare a favorable ground for repairing damaged NM [5]. Unfortunately, such multi-target treatments are not yet discovered.

The best preventive approach would be to avoid or to minimize allergen exposure but this approach is not practical because allergens may be outdoor or indoor and not visible to naked eye. Wearing a cotton or polyester mask, as was the case in 2020-21 to protect against Covid-19, is neither practical nor feasible without isolating the individuals from social interactions [30].

For many decades, researchers have been trying to find an alternative to cotton or polyester masks and to conceive a liquid mask which can directly be applied on the NM. Such liquids are presented as sprays containing saline solutions, sea water or essential oil with or without a thickening or jellifying ingredient such as plant gums, HPC or HPMC (Hydroxypropyl methyl cellulose), carrageenin, xanthan or acacia gum, pectin, starch, or other polysaccharide, usually applied as nasal sprays [1]. None of these filmogen solutions proved any real efficacy because during $\mathrm{AR}$, the nose is either dripping or occasionally blocked. Any liquid solution devoid of property to form a stable film on the NM and to resist physical / mechanical pressures exerted on the film, may not stay on the NM for more than a few minutes. Such solution or film gets diluted with nasal liquid flow and immediately loses all its nasal surface protective or allergen barrier properties. Recently, direct nasal inhalation of HPMC powder is suggested as a better alternative to liquid preparations. HPC or HPMC is spread in a nostril as a powder where it absorbs nasal liquid, swells, and forms a gel barrier against airborne environmental allergens and particulate matters [21]. Authors claim that such an HPMC film is safe and highly effective in preventing allergen contact with the NM but the duration of action and clinical efficacy are not mentioned [31]. We believe that any allergen protective nasal barrier should be filmogen, flexible, and resistant to mechanical pressures, at least for a few hours, to avoid repeated applications. Such a film should also be able to clean the NM of free-floating contaminants such as histamine, inflammatory cytokines, and cellular debris, which are already present or being generated on the NM to block inflammatory \& allergen cascades. In addition, such nasal barrier should be able to keep the NM clean, should be non-irritant, totally safe, should not have interactions with the underlying cells and should not be absorbed in the body to avoid sideeffects.

In the absence of such treatment or device, we envisaged conceiving a long-lasting (4-6h), absorbent, osmotic, glycerol based, polymeric liquid film, which can be applied on the NM as a spray to form a nasal mask.

Glycerol being osmotic and cell-friendly, we used it as a barrier and rendered it filmogen through polymeric binding. When sprayed on the $\mathrm{NM}$, such a stable film can block incoming allergenic particles and keep 
the NM clean by detaching and draining free-floating contaminants towards the film through osmotic liquid flow [25].

This article summarizes the conception of the film, its allergen trapping properties compared to HPMC in vitro, and its clinical efficacy in patients suffering from AR and pollution induced respiratory symptoms.

\section{Materials \& Methods}

\subsection{Conception of liquid filmogen glycerol bandage:}

1.1. Selection of osmotic filmogen ingredients: To find an ingredient which can be used to obtain a filmogen liquid for nasal application which is osmotic, non-cytotoxic, absorbent, and stable for 4-6h; multiple natural and synthetic ingredients were tested for osmotic and cytotoxic potential. The osmotic potential was quantified using multi-cellular live cell membrane dehydration model as described by Shrivastava et al (Ravi Shrivastava et al. 2021) and cytotoxicity potential was measured as per the NF EN ISO 10993 (Évaluation biologique des dispositifs médicaux Partie 5: essais concernant la cytotoxicité in vitro n.d.) [26]. Cellular irritation potential was quantified using in vitro Bovine Corneal Opacity Test (BCOP) as described by Schrage et al [23]. None of the ingredients met all the criteria except for glycerol which was osmotic and cell friendly but was slightly irritant and poorly filmogen.

1.2. Reducing irritation potential and improving absorbent capacity of osmotic glycerol: A few commonly used food-grade thickenings agents were added in the selected osmotic ingredient solution at different concentrations to introduce absorbent properties in the film. Only those thickening/jellifying agents which were safe and had no effects on glycerol osmosis were retained.

1.3. Selection of natural or synthetic polymer or polymeric associations to render glycerol film resistant and flexible: Glycerol associated with jellifying agents was an excellent osmotic solution. However, when applied on a live biological membrane such as the NM, the osmotic activity generated by the film created a strong hypotonic liquid flow from the mucosa towards the film, leading to instant dilution of the film and loss of osmotic activity within a few minutes. As certain inert and big polymers (ex. plant tannins or synthetic polymers) are known to bind with selected macromolecules ( $\mathrm{H}, \mathrm{OH}$ binding) and specific proteins (Vieira et al. 2011), after pre-screening, a few plant extracts, rich in glycerol molecule binding polymers, were retained to render the glycerol film stable to mechanical pressures [32].

1.4. Conception of filmogen glycerol osmotic film: The finished filmogen bandage contained glycerol, HPC, and glycerol binding plant polymeric mix in water with very small quantities of preservatives and technical ingredients. The proportions of glycerol and plant polymers were varied as per the site of application, required osmotic potential, absorbent capacity, flexibility, and resistance of the film.

\subsection{Evaluation of allergen preventive barrier properties of glycerol film}

2.1. Test allergens: To evaluate allergen prevention barrier properties of the polymeric glycerol film (PCNS), we used Amb a 1 (ambrosia, ragweed), Der $\mathrm{p} 1$ and 2 (dust mite), Alt a 1 (Alternaria, fungus), and Fel d 1 (cat) allergens, representing common indoor and outdoor allergens. The allergens and ELISA test plates to test and to quantify allergen antigens were purchased from INDOOR biotechnologies (Cardiff, UK) and the tests were performed as per the instruction manual of the supplier. For the allergen diffusion tests, all allergens were used at a concentration of $5 \mu \mathrm{g} / \mathrm{ml}$ in PBS. Powdered HPMC was used as a positive comparator product.

2.2. Test product concentrations: PCNS was presented as a liquid solution in a 15-ml plastic vials. As the volume of PCNS administered in each nostril is between $120 \mu \mathrm{l}$ ( 1 spray) or $240 \mu \mathrm{l}$ ( 2 sprays), the same volumes were selected for PCNS and HPMC to form an allergen barrier for in vitro testing.

The positive control HPMC was purchased from Lake Consumer Product Inc., USA (a subsidiary of Wisconsin Pharmacal Company, I Pharmacal Way, Jackson, WI 55037, USA). The product is sold as a powder spray for nasal application ( $800 \mathrm{mg}$ HPMC for 200 sprays, 1 spray per nostril).

The dose of HPMC per application / nostril in the comparator product is $4 \mathrm{mg}$ powder. Therefore, to reproduce identical concentration for the immunological test, a test solution of $4 \mathrm{mg} \mathrm{HPMC} / \mathrm{ml}$ was prepared for testing. PBS (Phosphate Buffer Saline) was used as a negative control. The test volumes $(120 \mu \mathrm{l}$ and $240 \mu \mathrm{l} /$ test $)$ were kept identical to PCNS volumes tested. All tests were performed in duplicate $(n=3$ each concentration).

2.3 Allergen transport through the film: To evaluate allergen penetration through the filmogen barrier of HPMC or PCNS compared to PBS film, the test products were placed as a film on the agar and the allergens were poured on the film. The aim was to measure the quantity of allergen capable to penetrate the test product film and reach up to the agar.

2.4 Allergen diffusion testing method: The tests were performed in 2-ml microtubes. Required microtubes were pre-labelled and $500 \mu \mathrm{l}$ of an agar solution $\left(1.5 \%\right.$ in $\mathrm{PBS}$ at $\left.57^{\circ} \mathrm{c}\right)$ was introduced. After $30 \mathrm{~min}, 120$ or 240 $\mu 1$ of each test or control solution (PCNS pure, HPMC $0.40 \%$, or PBS control) were then gently poured in the tube on the agar layer and allowed to settle for 5 -minutes. $20 \mu \mathrm{l}$ of respective allergen solution $(5 \mu \mathrm{g} / \mathrm{ml}$ of total $100 \mu \mathrm{g}$ ) was then deposited on the test product layer in the respective tubes. The microtubes were then incubated at $37^{\circ} \mathrm{C}$ for $15,30,60$, and 120 min for the $1^{\text {st }} \mathrm{Amb}$ a 1 allergen to select two optimal allergen diffusion times and thereafter for 30 and 90-min for all other allergens. After the incubation period, the microtubes were washed twice with 1xPBS by slowly filling and emptying the tubes to remove allergen and test solutions. Agar was then removed with a spatula, introduced in a microtube containing 1-ml PBS Tw0.1\% and vortexed. Baseline control allergen controls were prepared ( $\mathrm{n}=2$ each) by adding $20 \mu \mathrm{l}$ of $5 \mu \mathrm{g} / \mathrm{ml}$ allergen solution in a microtube without agar. All the microtubes were kept overnight at $+4{ }^{\circ} \mathrm{C}$ under slow agitation. The next day, the microtubes were removed, brought to room temperature, vortexed, and centrifuged for 5-min. (5000 RPM/min). The supernatants were then analysed to quantify the concentration of allergen crossing the test product film and infiltrating into the gel, through ELISA kits, for each allergen.

2.5 ELISA tests to quantify allergens in the agar: 2-plates were used for each test ( $\mathrm{n}=4$ wells/test solution). All tests were performed at room temperature as described by the supplier. In short, the ELISA plates were washed thrice with $150 \mu \mathrm{l}$ wash buffer followed by deposits of $100 \mu \mathrm{l} /$ well of test samples, standards, or blank (dilution buffer). The plates were then incubated for $1 \mathrm{~h}$ and washed again with wash buffer, followed by depositing $100 \mu \mathrm{l}$ of the $1: 1000$ diluted conjugate mixture with $1: 1000$ diluted streptavidin-HRP/well as indicated by the test supplier. The plates were incubated for $1 \mathrm{~h}$, washed with $150 \mu \mathrm{l}$ wash buffer followed by adding $100 \mu \mathrm{l}$ of TMB (3,3',5,5'-Tetramethylbenzidine) / well. After 4min incubation, the reaction was terminated by adding $50 \mu 1$ stop solution per well. Optical densities were measured at 450nm.

Result interpretation: The mean concentration of allergens present in the agar gel was quantified in $\mathrm{ng} / \mathrm{ml}$ of sample. The mean baseline value obtained with PBS as a preventive barrier for each allergen was considered as the baseline value (100\% concentration). The mean allergen concentrations in HPMC and PCNS barrier were expressed as \% compared to baseline values.

\subsection{Clinical efficacy evaluation:}


3.1. Type of study performed: An observational, parallel-group, comparative, randomized, placebo controlled, clinical trial was performed to evaluate efficacy and safety of PCNS versus placebo $(0.9 \% \mathrm{NaCl}$ saline solution spray) in patients suffering from allergen and/or pollution induced rhinitis.

3.2. Study organizer: The clinical part was conducted at Nexus Clinical Research Center in India, affiliated to Nexus Clinical Research LLC, USA. The protocol and study design were approved by the Institutional Ethical Committee of India - Rajiv Gandhi Institute of medical sciences (EC Registration $\mathrm{N}^{\circ}$ ECR/492/Inst/AP/2013, dated 05/12/2013) and the trial was conducted following the ICH-GCP guidelines as per the declaration of Helsinki concerning ethical principles for medical research for a topically applied medical device involving human subjects.

3.3. Study population: Male and female patients aged between 8 and 65 years, having history of allergy and clinical respiratory and/or AR symptoms. It was planned to enroll at least 30 patients in TP group and 15 in $\mathrm{CP}$ group to obtain statistically significant data. Less patients were taken in the saline solution treated control group as use of saline solution for the treatment of AR is well documented and its clinical efficacy is well known.

3.4. Inclusion and exclusion criteria: At the time of recruitment at the study centers, patients were examined physically, and patient's medical, surgical, allergic and respiratory symptom history was checked and recorded. Vital signs such as blood pressure, pulse rate, and respiratory rate were measured. Patients not suffering from any serious pathology and having a history of at least 2-year acute seasonal or perennial hay fever and/or or pollution induced AR symptoms were then examined for enrollment in the study. The main inclusion criteria comprised: ready to abstain from all anti-allergic medication or any other treatment which may affect study outcome (except in the case of strict necessity where such treatment was prescribed by the clinical investigator), patients having mean daily reflective Total Nasal Symptom Score (rTNSS) of $\geq 3$ on a scale of 6 and mean daily Reflective Ocular Symptom Score (rTOSS) of $\geq 2$ on a scale of 4 for the previous 7-days (day -6 to 0 ). Key exclusion criteria included: hypersensitivity to any of the investigational product's components, chronic disabling diseases, or being under any treatment which may affect the study outcome.

3.5. Randomization: After screening, patients satisfying all the inclusion criteria were enrolled and randomly allocated, in a 2:1 ratio, to either test product (TP) or comparator product (CP) groups. Randomization was performed using SAS Version 9.1.3 following a randomization schedule. Block Randomization methodology was employed for generating the list. Within the block the treatments were distributed in the ratio of 2:1. Each patient received a unique screening identification number, randomization code, and enrollment identification number and a personal diary for daily recording.

3.6. Product presentation and administration: TPs and CPs were supplied by Vitrobio, France (Issoire) and were presented identically (15 ml spray containing a slightly viscous and colorless liquid) except for the product code and the batch number. The TP contained PCNS solution while CP contained $0.9 \% \mathrm{NaCl}$ saline solution.

3.7. Product application: Clinically affected patients were asked to apply 2-3 pulverizations in each nostril, 3 to 4 times per day (morning, mid-day, evening, and before night rest). As a preventive measure, patients were asked use 1-2 sprays in each nostril, 15-min before anticipated pollution / allergen exposure. Patients were included in the study on day -6 , started treatment on day 1 and finished the treatment course on day 22.

\subsection{Parameters recorded:}

Patients were asked to record the symptoms in the morning (AM) or evening (PM), just prior to dosing (instantaneous rating, [i]) and over the previous 12 hours (reflective rating, [r]) for the nasal, ocular and respiratory symptoms.

Daily scores of rhinorrhea, congestion, sneezing, and itching were recorded for each patient and mean values of these 4 parameters were calculated to obtain reflective Total Nasal Symptom Score (rTNSS) whereas three ocular symptoms (eye itching, tearing, and eye redness) were combined to calculate reflective Total Ocular Symptom Score (rTOSS). The instantaneous pre-dose morning ocular scores were summed to obtain an instantaneous pre-dose TOSS (am-pre-dose iTOSS). The mean of results at an interval of 6-days was calculated and analyzed.

The scores of rhino-conjunctivitis qualities of life (RQLQ) were also evaluated at the start and at the end of treatment. As proposed by Juniper et al, 1991 (Juniper and Guyatt 1991), the RQLQ had 28 questions in 7 domains (activity limitation, nasal symptoms, eye symptoms, nonnose/eye symptoms, practical problems, and emotional function). Patients recall how bothered they have been by their rhino-conjunctivitis at the start $\&$ at the end of the treatment on a 7-point scale $(0=$ not impaired at all, $6=$ severely impaired). The overall RQLQ score represent mean of 28 responses and the individual domain scores are the means of items in those domains. Mean difference compared to baseline and controls, at the start and at the end of the treatments, were evaluated.

In addition, the need for rescue medicine (RM) was also calculated daily on a four-point scale $(0=$ no rhinitis medicine; $1=$ cetirizine, $10 \mathrm{mg} / \mathrm{d}$, $2=$ cetirizine, $20 \mathrm{mg} / \mathrm{d}, 3=$ systemic or topical corticosteroids for AR). The mean scores in each group were evaluated. When more than one RM was used on the same day, only the maximal medication score was recorded.

\subsection{Statistical analysis of results:}

The demographic details were calculated using Wilcoxon rank sum test. All the analyses were carried out on as "intent-to-treat" basis with Microsoft Excel and XLStat using the available data. Significant effects were those with a probability lower than $\alpha=0.05$. For each score, repeated measures analysis of variance (RMANOVA) determined differences in symptoms scores across study visits. Nemenyi post-hoc test provided pairwise comparisons in a group between baseline and the end of the study. Results were compared with baseline values (before $1^{\text {st }}$ treatment on day 1) in the same group and between the groups, at each time point.

\subsection{Results}

6.1. Conception of PCNS filmogen bandage: PCNS formulation contained Glycerol with Hydroxypropylcellulose (3.55\%), polymeric mix derived from the plant extracts of Vaccinium myrtillus, Hedera helix, and Curcuma longa $(<1.0 \%)$ with very small quantities of Mentha piperita essential oil, Sodium Benzoate, Potassium Sorbate, Citric Acid in water [27]. When spread on the NM (120-240 $\mu 1 /$ spray/nostril), the liquid instantly forms an osmotic, absorbent, and non-irritant protective film.

6.2. Safety, stability and osmotic properties of the film: The in vitro and in vivo cytotoxicity tests and 28-day, repeated dose, nasal application studies in rats showed no, local or systemic adverse effects. Exposing PCNS filmogen liquid on the in vitro NM mimicking multicellular live cell membrane showed that PCNS film is resistant to mechanical pressure, remains on NM for 4-6h, the osmotic activity continues during this period to attract and to trap free-floating NM contaminants towards PCNS film [26].

\subsection{Allergen barrier forming properties:}

Test product volume and incubation period finding study: Three volumes (40, 120, and $240 \mu \mathrm{l})$ of Amb a 1 allergen were incubated for 15, 30, 60, and $120 \mathrm{~min}$. Amb a 1 concentration penetrating through the test product film and reaching up to the gel was measured by ELISA. 
In table 1 , Results show that a test product volume of $40 \mu 1$ forms a very thin film for controls and all the test products, as the quantity of allergen in the gel was not significantly different in the test product groups compared to controls indicating that allergen can easily cross the barrier. When test product volumes were $120 \mu \mathrm{l}$ and $240 \mu \mathrm{l}$, a volume dependent slight reduction of allergen concentration in agar gel was observed for PBS and $0.4 \%$ HPMC but the concentration was comparable. On the contrary, PCNS nearly totally blocked allergen diffusion up to the agar gel ( $<2.6 \%$ detection limit) after 15, 30 and 60 min of incubation. At 120 min, the Amb a 1 concentration was only $8 \%$ and $4 \%$ compared to baseline with $120 \mu 1$ and $240 \mu$ l, respectively. These findings clearly show that PCNS film effectively prevents the entry of Ragweed Amb a 1 allergen while PBS and HPMC have no effect.

\begin{tabular}{|c|c|c|c|}
\hline $\mathbf{S ~ N}^{\circ}$ & Symptom & Test product (Total $\mathbf{n = 2 9})$ & Comparator saline group $(\mathbf{n}=\mathbf{1 7})$ \\
\hline 1 & Wheezing & $17(58.62 \%)$ & $11(64.70 \%)$ \\
\hline 2 & Chest tightness & $22(75.86 \%)$ & $9(52.94 \%)$ \\
\hline 3 & Cough & $20(68.96 \%)$ & $12(70.58 \%)$ \\
\hline 4 & Dyspnea & $63.75 \%$ & $61.76 \%$ \\
\hline \multicolumn{2}{|c|}{$\begin{array}{l}\text { Mean population with } \\
\text { respiratory symptoms }\end{array}$} & & \\
\hline
\end{tabular}

Table 1: Results are expressed as mean \% allergen detected in gel compared to baseline controls. < indicates the limit of detection for that allergen.

Based on this initial minimum test volume and incubation period finding study, further experiments were performed only with $120 \mu 1$ and $240 \mu 1$ volume barriers incubated with the allergens for 30 and $90 \mathrm{~min}$. In the microtube, all the test products $(120$ and $240 \mu 1)$ were placed on a layer of agar $(0.5 \mathrm{ml})$ and the allergen solution $(20 \mu \mathrm{l}$ containing $5 \mu \mathrm{g} / \mathrm{ml}$ of each allergen) was spread on top of the product films. (Table 2)

\begin{tabular}{|c|c|c|c|c|c|c|c|c|c|}
\hline \multirow[t]{2}{*}{$\begin{array}{c}\text { Allergen } \\
\text { tested : }\end{array}$} & \multirow{2}{*}{$\begin{array}{c}\begin{array}{c}\text { Incubation time (in } \\
\text { min) à }\end{array} \\
\text { Test solution }\end{array}$} & \multicolumn{2}{|c|}{ Plate $130 \mathrm{~min}$} & \multicolumn{2}{|c|}{ Plate $190 \mathrm{~min}$} & \multicolumn{2}{|c|}{ Plate $230 \mathrm{~min}$} & \multicolumn{2}{|c|}{ Plate $290 \mathrm{~min}$} \\
\hline & & $\begin{array}{l}120 \\
\mu \mathrm{L}\end{array}$ & $\begin{array}{l}240 \\
\mu \mathrm{L}\end{array}$ & $\begin{array}{l}120 \\
\mu \mathrm{L}\end{array}$ & $\begin{array}{l}240 \\
\mu \mathrm{L}\end{array}$ & $\begin{array}{l}120 \\
\mu \mathrm{L}\end{array}$ & $\begin{array}{l}240 \\
\mu \mathrm{L}\end{array}$ & $\begin{array}{l}120 \\
\mu \mathrm{L}\end{array}$ & $\begin{array}{l}240 \\
\mu \mathrm{L}\end{array}$ \\
\hline \multirow[t]{3}{*}{ Amb a 1} & PBS control & $10 \%$ & -OR- & $20 \%$ & $13 \%$ & $9 \%$ & $4 \%$ & $17 \%$ & $11 \%$ \\
\hline & Allerblock $0,4 \%$ & $17 \%$ & $9 \%$ & $20 \%$ & $16 \%$ & $14 \%$ & $9 \%$ & $17 \%$ & $14 \%$ \\
\hline & PCNS & $<3 \%$ & $<3 \%$ & $<3 \%$ & $<3 \%$ & $<3 \%$ & $<3 \%$ & $<3 \%$ & $4 \%$ \\
\hline \multirow[t]{3}{*}{ Der p 1} & PBS control & $9 \%$ & $6 \%$ & $17 \%$ & $13 \%$ & $10 \%$ & $6 \%$ & $16 \%$ & $13 \%$ \\
\hline & Allerblock $0,4 \%$ & $12 \%$ & $10 \%$ & $17 \%$ & $12 \%$ & $12 \%$ & $10 \%$ & $18 \%$ & $12 \%$ \\
\hline & PCNS & $<2 \%$ & $4 \%$ & $4 \%$ & $5 \%$ & $<2 \%$ & $4 \%$ & $4 \%$ & $5 \%$ \\
\hline \multirow[t]{3}{*}{ Der p 2} & PBS control & $23 \%$ & $14 \%$ & $31 \%$ & $18 \%$ & $21 \%$ & $12 \%$ & $29 \%$ & $16 \%$ \\
\hline & Allerblock $0,4 \%$ & $22 \%$ & $17 \%$ & $26 \%$ & $21 \%$ & $20 \%$ & $16 \%$ & $25 \%$ & $19 \%$ \\
\hline & PCNS & $<4 \%$ & $<4 \%$ & $8 \%$ & $<4 \%$ & $<4 \%$ & $<4 \%$ & $8 \%$ & $<4 \%$ \\
\hline \multirow[t]{3}{*}{ Alt a 1} & PBS control & $10 \%$ & $6 \%$ & $15 \%$ & $9 \%$ & $13 \%$ & $8 \%$ & $19 \%$ & $12 \%$ \\
\hline & Allerblock $0,4 \%$ & $6 \%$ & $7 \%$ & $9 \%$ & $6 \%$ & $8 \%$ & $9 \%$ & $12 \%$ & $8 \%$ \\
\hline & PCNS & $3 \%$ & $3 \%$ & $6 \%$ & $6 \%$ & $4 \%$ & $4 \%$ & $7 \%$ & $7 \%$ \\
\hline \multirow[t]{3}{*}{ Fel d 1} & PBS control & $5 \%$ & $4 \%$ & $6 \%$ & $4 \%$ & $6 \%$ & $4 \%$ & $7 \%$ & $5 \%$ \\
\hline & Allerblock $0,4 \%$ & $3 \%$ & $4 \%$ & $2 \%$ & $3 \%$ & $3 \%$ & $4 \%$ & $2 \%$ & $3 \%$ \\
\hline & PCNS & $<1 \%$ & $2 \%$ & $2 \%$ & $3 \%$ & $<1 \%$ & $2 \%$ & $2 \%$ & $3 \%$ \\
\hline $\begin{array}{l}\text { OR : outlier } \\
\text { result }\end{array}$ & & & & & & & & & \\
\hline
\end{tabular}

Table 2: Amb A1(Ragweed allergen), Der $p 1$ (dust mite), Bet v 1 (Birch), Der $p 2$ (dust, mite), Alt a 1 (Alternaria, fungus allergen), and Fel d 1 (cat dander) allergens were exposed on the test product or PBS solutions and the concentration of allergens in the gel was measured at 30 and 90 min. Results are expressed as \% mean allergen concentration ( $n-3$, repeated twice) in the gel compared to the concentration of the same allergen detected in

Amb a 1: For PBS and HPMC 0.4\%, Amb a 1 allergen diffusion was lower with $240 \mu \mathrm{l}$ barrier compared to $120 \mu \mathrm{l}$ but the differences were not significant. PCNS in both volumes was very effective in inhibiting the diffusion of ragweed Amb a 1 allergen as the mean concentrations in the agar gel were $<3 \%$ or $4 \%$ compared to baseline in both volumes at 30 and 120 min.

Der $\mathrm{p}$ 1: In this experiment, surprisingly the concentration of Der $\mathrm{p} 1$ detected in PBS tubes at $30 \mathrm{~min}$ (both plates) was between 6-10\% compared to $10-12 \%$ in HPMC film tubes. This difference is not highly significant but shows that neither PBS nor HPMC solutions constitute an effective barrier against Der $\mathrm{p} 1$ allergen at the concentrations used in this study. The concentration of this allergen in PCNS layered tubes was 5\% or below $5 \%$ at 30 and $90 \mathrm{~min}$ in both plates. These results confirm the findings that PCNS is a good filmogen barrier against dust mite allergens.

Der $\mathrm{p} 2$ : This is a different but common indoor mite dust allergen. When exposed on PBS solution layer, the mean concentration reaching up to the 
agar gel with 120 and $240 \mu \mathrm{l}$ barrier after 30 and 90 min was $20.50 \%$ compared to baseline while it was $20.75 \%$ for HPMC. The concentrations were higher in $120 \mu 1$ layers compared to $240 \mu 1$. In the PCNS group, the mean concentration at all-time points was $5.0 \%$ or below, right after 30 min of incubation indicating that PCNS forms a good preventive barrier against this allergen.

Alt a 1 - Alternaria, fungus allergen: In the PBS controls, about $15 \%$ allergen reached up to the agar gel layer after 30 or $90 \mathrm{~min}$. Slightly higher values were observed at $90 \mathrm{v} / \mathrm{s} 30 \mathrm{~min}$. HPMC formed a better allergen barrier to prevent the diffusion of this allergen compared to PBS, as on an average, only $8.12 \%$ of allergen was detected in the agar gel. In the PCNS group also, the allergen diffusion was hampered as the mean value of all the time points and both volumes were $5.0 \%$, showing less diffusion compared to $0.4 \%$ HPMC.

Fel d 1: This allergen is light and remains suspended on the surface of the barrier film. In PBS controls, only 4 to $7 \%$ (mean 5.1\%) was detected in the agar gel for both volumes and at $30 \& 60$ minutes of incubation. The percentage of allergen found in HPMC was only between 2 to $4 \%$ (mean 3.375 ) with a mean reduction of $33.82 \%$ compared to PBS indicating that $0.4 \%$ HPMC effectively reduces allergen diffusion up to the agar gel by forming a preventive barrier. PCNS was even more effective as allergen concentration was between $<1$ to $3 \%$ (mean $2.0 \%$ or a reduction of $60.78 \%$ compared to PBS), twice as high compared to $0.4 \%$ HPMC.

The results on allergen prevention barrier effects show that PCNS forms a strong barrier against all the allergens tested compared to HPMC, which forms a moderate and probably selective barrier against the allergens tested in this study.

\subsection{Clinical efficacy:}

6.4.1. Population analyses: In this observational study, 49 patients were randomized, 32 were included in test product group and 17 in control group (saline solution spray), 3 patients in PCNS group and 2 in the control group did not complete the study for reasons not related to the test products. At the end of the study, 29 patients in test product (24M and 5F) and $15(14 \mathrm{M}$ and $1 \mathrm{~F})$ in the saline solution treated group were analysed.
6.4.2. AR symptoms analysed: The presence/severity of four nasal (rhinorrhoea, nasal congestion, sneezing, itching), three ocular (eye itching/burning, eye tearing/watering, and eye redness), and four respiratory symptoms (wheezing, chest tightness, cough, and dyspnoea) was recorded in the morning just prior to dosing (instantaneous rating, [i]) and over the previous 12 hours (reflective rating, $[\mathrm{r}]$ ). A reflective assessment for the previous 12 hours was also conducted in the evening (PM). The three individual reflective nasal and ocular symptom scores were combined to obtain an AM and PM reflective total nasal symptom score (AM rTNSS, PM rTNSS) and total ocular symptom score (AM rTOSS, PM rTOSS), respectively. Similarly, the instantaneous pre-dose morning scores were summed to obtain an instantaneous pre-dose TOSS (AM pre-dose iTOSS). All the scores were recorded on a 0-3 scoring scale during the study $(0=$ none, $3=$ severe $)$.

These two scores were averaged to obtain a daily reflective total nasal (daily rTNSS), ocular (daily rTOSS), and respiratory symptom scores.

As the mean scores of instant symptoms for all the parameters are closely linked to the observations of reflective (r) symptom scores, only the results of key parameters and mean total reflective symptom scores are presented.

6.4.3. Analyses of total nasal symptoms: The key nasal symptoms in AR are rhinorrhea and nasal congestion.

As shown in (Figure. 1-a), in the placebo saline spray treated group, the 6-day interval mean score for rhinorrhea was $1.88( \pm 0.70)$ and 2.24 $( \pm 0.75)$ on day 1 (baseline), at the start of treatment. After treatment initiation, symptoms aggravated slightly up to day 6 (mean score 2.44 \pm 0.56 ) but started reducing thereafter progressively up to day 22 (mean score $1.29 \pm 0.59$ ). In the PCNS treated group, rhinorrhea was strong during the $1^{\text {st }} 2$ days of treatment but showed a reduction of $36 \%, 54 \%$, $69 \%$, and $79 \%$ on days $6,12,18$, and 22 , respectively compared to day 1 . The reduction was statistically significant $(\mathrm{p}<0.001)$ compared to controls and compared to day 1 for all the time points. High rhinorrhea in the PCNS group during the $1^{\text {st }}$ few days of treatment may have been related to the osmotic properties of the test product on a damaged NM.

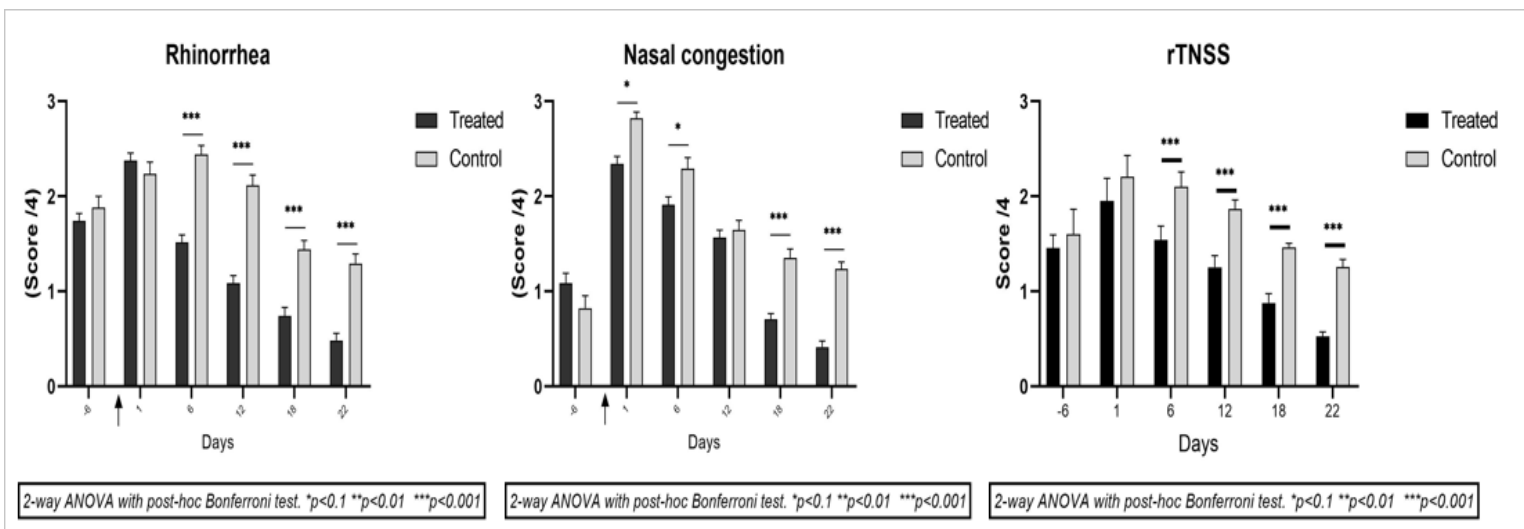

Figure 1: Mean nasal symptom intensity of rhinorrhoea (a), nasal congestion (b), and reflective mean total nasal symptom scores of rhinorrhoeas, sneezing, nasal congestion, and itching (c-rTNSS) of 29 patients in the PCNS treated (black lines) and 15 in the comparator control (gray lines) group. The mean scores of PCNS group were compared with the control on days 6, 12, 18, and 22 of treatment. * Indicates statistical difference $v / s$ controls.

The mean nasal congestion score (Figure. 1 - $b$ ) in the placebo saline group at baseline was $2.82( \pm 0.39)$ which varied only slightly after initiation of the treatment and decreased down to $2.29( \pm 0.65)$ on day 6 and 1.65
$( \pm 0.60)$ on day 12 , and $1.35( \pm 0.55)$ on day 18 . The decrease was nearly $50 \%$ compared to baseline from day 18 onwards. 


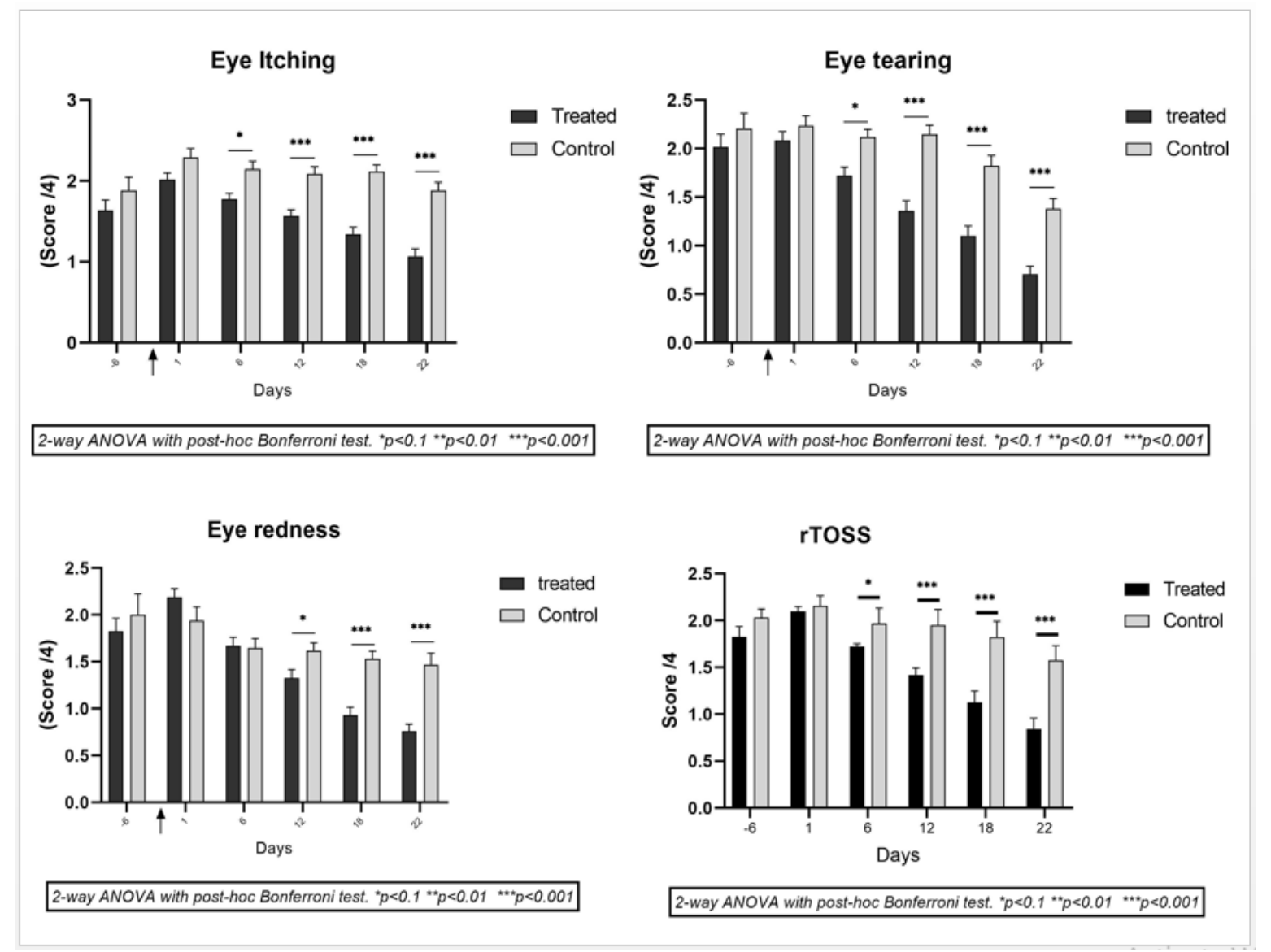

Figure 2: Mean ocular symptom intensity of eye itching $(a)$, eye tearing $(b)$, eye redness $(c)$ and reflective mean total ocular symptom scores $(r T O S S$ d) of 29 patients in the PCNS treated (black lines) and 15 in the comparator control (gray lines) group. The mean scores of PCNS group were compared with the control on days $6,12,18$, and 22 of treatment. *Indicates statistical difference $v / s$ controls.

In the PCNS group, the initial baseline mean score of 2.34 decreased slowly and progressively to $1.91,1.57,0.71$, and 0.41 on days $6,12,18$, and 24 , showing a reduction of nasal congestion by $18 \%, 33 \%, 70 \%$, and $82 \%$, respectively over 22 days. The difference was statistically significant $\mathrm{v} / \mathrm{s}$ comparator, $\mathrm{p}<0.001$ from day 18 onwards.

Same as that of rhinorrhea and nasal congestion, sneezing and itching symptoms also slowly decreased until the end of the treatment period in both the groups, but the reduction was nearly two times faster in the PCNS v/s comparator group.
The mean values of the 4 nasal parameters (rTNSS - Figure. 3-c) evaluated by the patients and the investigator at an interval of 6 days show that the mean nasal symptom scores were relatively strong in both groups at the start of the study (T0 or baseline). In the saline treated group, the mean of total nasal symptom scores showed a tendency towards reduction during the first 12 days compared to baseline values, but the improvement was faster thereafter. In the PCNS group, the mean nasal symptom scores started decreasing right after the first week of treatment up to the end of the study. This reduction was nearly two times faster in this group with statistically significant $(\mathrm{p}<0.001)$ reduction $\mathrm{v} / \mathrm{s}$ control. 


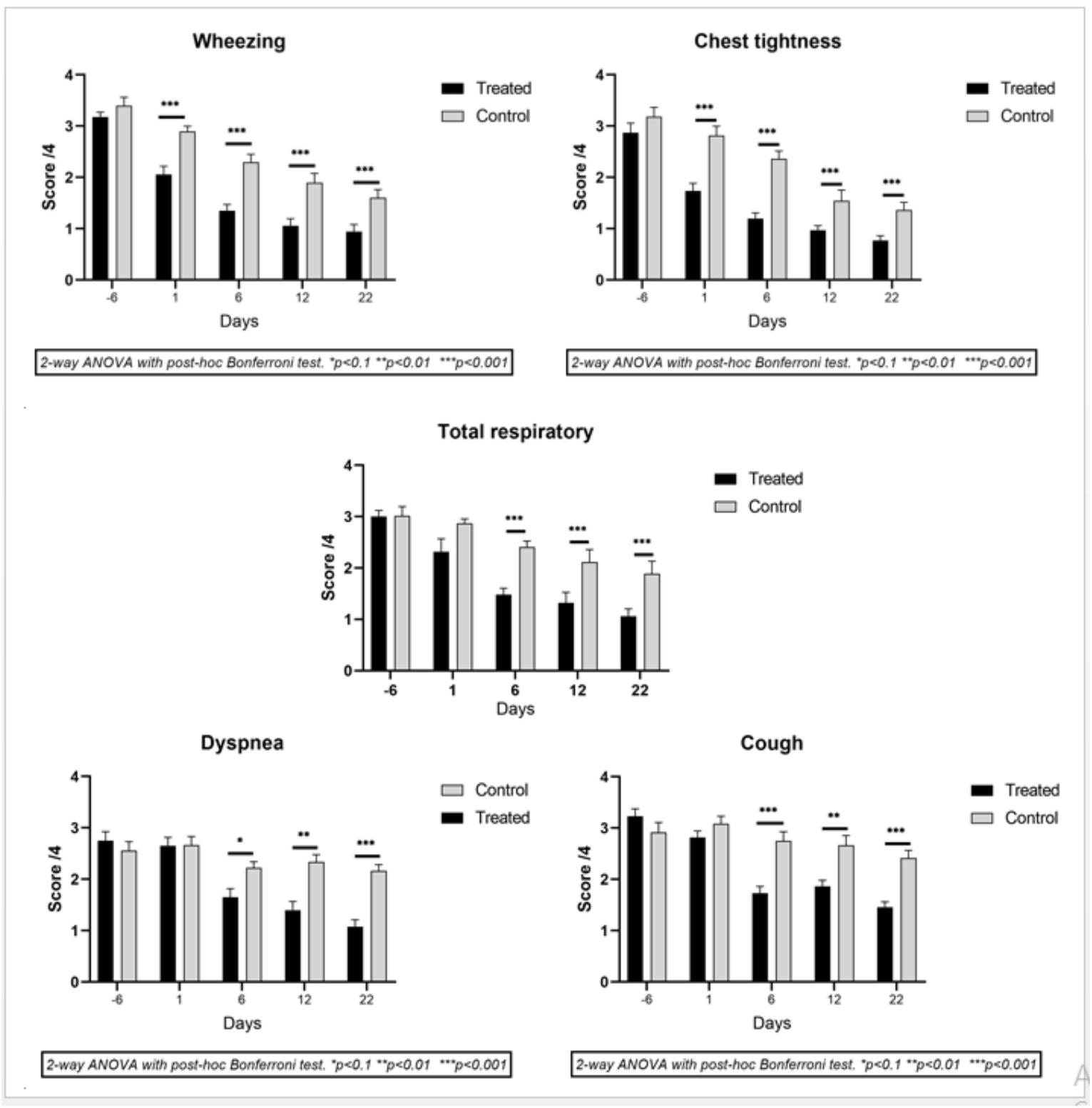

Figure 3: Mean respiratory symptom intensity of wheezing, feeling of chest tightness, intensity and frequency of cough, and dyspnoea and total mean respiratory symptom scores of these parameters of patients in the PCNS treated (black lines) and in the comparator control (Gray lines) group showing these symptoms. The number of patients having these symptoms varied during the study. The mean scores of PCNS group were compared with the control on days $6,12,18$, and 22 of treatment. * Indicates statistical difference $\mathrm{v} / \mathrm{s}$ controls.

The mean TNSS scores also followed the same pattern.

\subsubsection{Ocular Symptoms:}

In AR, ocular symptoms are generally concomitant with nasal symptoms.

In PCNS group, the mean rTOSS score (the mean of the 3 ocular symptom scores) at baseline week was 2.10 which reduced to $1.72( \pm 0.52)$ on day $6,1.42( \pm 0.75)$ on day $12,1.13( \pm 0.52)$ on day 18 , and $0.84( \pm 0.65)$ on day 22. These scores show progressive reduction of allergic eye symptoms by $17.8,32.3 \%, 46.3 \%$, and $59.7 \%$ on days $6,12,18$, and 22 in PCNS group v/s $8.6 \%, 9.5 \%, 15.4 \%$, and $26.8 \%$ in comparator group over the same period.

When the results of both groups are compared, the reduction in ocular symptom is much faster in the test product treated patients compared to the corresponding reduction observed in the placebo group. Compared to controls, the mean rTOSS scores were statistically significant $(\mathrm{p}<0.1$ on day 6 and $<0.001$ onwards) for PCNS.

6.4.5. Respiratory Symptoms: Number of patients having respiratory symptom at the time of randomization in each group is shown in the (Table 3). 


\begin{tabular}{|c|c|r|r|}
\hline S N & Symptom & Test product(Total n=29) & Comparator saline group(n=17) \\
\hline 1 & Wheezing & $17(58.62 \%)$ & $10(58.82 \%)$ \\
\hline 2 & Chest tightness & $15(51.72 \%)$ & $11(64.70 \%)$ \\
\hline 3 & Cough & $22(75.86 \%)$ & $12(70.58 \%)$ \\
\hline 4 & Dyspnea & $20(68.96 \%)$ & $61.76 \%$ \\
\hline $\begin{array}{l}\text { Mean population with } \\
\text { respiratory symptoms }\end{array}$ & $63.75 \%$ & \\
\hline
\end{tabular}

Table 3: The number of patients with one or more respiratory symptoms in each group.

Each symptom was scored as 0 (no symptom), 1 (mild,) 2 (moderate), 3 (severe) or 4 (very severe) and mean respiratory symptoms scores are shown in Figure 3.

This study was performed in congested city environment in people allergic to city pollution and environmental allergens and about $60 \%$ patients in each group suffering from AR had concomitant respiratory symptoms. Results show that the incidence of all four respiratory symptoms (wheezing, cough, dyspnea, and feeling of chest tightness) was progressively decreased in both groups but the effects were much faster and stronger with PCNS treatment. At the end of the study (day 22), mean respiratory symptoms were reduced by $15.39 \%$ in controls compared to $40.49 \%$ in the PCNS group ( $\mathrm{p}<0.001 \mathrm{v} / \mathrm{s}$ control from day 6 onwards).

6.4.6. Nasal smear: Repeated exposure to nasal allergens leads to longterm changes in local and systemic inflammation, including up-regulation of circulating eosinophils and allergen-specific $\operatorname{IgE}$ and enhanced systemic response to allergens.

In the control group, the mean baseline eosinophil count was 19.13 $( \pm 5.34)$ which reduced to $11.17( \pm 4.21)$ on day 22 while in PCNS group the initial baseline count of $14.95( \pm 6.20)$ reached $4.33( \pm 1.62)$ at the end of the study. The reduction in eosinophil count is largely due to the cleaning effects of saline solution and the test product. The test product being slightly hypertonic and osmotic, attracts contaminants, including eosinophils present on the nasal mucosa towards to absorbent PCNS film.

6.4.7. RQLQ: The mean scores of 28 questions in 7 domains related to effect on daily activity, sleep, nose/eye symptoms, practical problem, nasal symptoms, eye symptoms, and emotions was evaluated on a 0-7 scale. Lower scores indicate better RQLQ. The baseline values were $13.87( \pm 2.31)$ in the control and $13.195( \pm 2.03)$ in the PCNS group. At the end of the study the mean scores were $10.675( \pm 1.72)$ in the control group v/s $7.04( \pm 2.06)$ in the PCNS group. This shows an improvement of $23.07 \%$ in controls and $46.65 \%$ in PCNS treated group.

Rescue medicine: $1 / 29$ patients in the PCNS group on day 15 and 4/15 in the control group during the $2^{\text {nd }}$ week of treatment took RM showing that reduction in AR symptoms with PCNS helps to minimize the need for acute antihistamine medications.

\section{Discussion:}

Allergic rhinitis (AR), caused by immunoglobulin E (IgE)-mediated reactions to inhaled allergens is becoming one of the most common chronic upper respiratory condition in the world [7]. The prevalence of AR is estimated above $30 \%$ in congested cities. Epidemiological studies show exacerbation of AR symptoms with co-exposure of allergen and air pollutants [29]. This explosive association induces nasal mucosa damage,
AR symptomatic intensity, and enhanced inflammatory response with the recruitment of inflammatory cells, cytokines, and interleukins. AR induced inflammatory cascade further leads to respiratory diseases such as asthma [11]. The disease become chronic and multi-factorial and cannot be treated with a single chemical drug. All the currently available treatments such as antihistamines, decongestants, corticosteroids, mast cell stabilizers, leukotriene inhibitors, allergen immunotherapy, and epinephrine shots, are of chemical origin and their long-term use causes significant side effects [19]. Moreover, none of these remedies is multifactorial and has any preventive effects. Complete avoidance of allergens and air pollution remains the best solution, but it does not form a total allergen barrier and is not always feasible except for permanent use of cotton or polyester masks which is not practical. Therefore, the aim of our research was to find an osmotic, liquid, bandage which can be applied directly on the NM and which remains stable for a period of 4-6h or more. We rendered glycerol filmogen and stable by trapping free glycerol molecules with specific glycerol binding polymeric structures to improve the film resistance to mechanical forces to which it is exposed on the NM. Thickening ingredients were incorporated in the film to enhance its absorbance capacity without being irritant to the NM. Such a film can be used to avoid NM contact with any contaminant in the air, whether it's a virus, bacteria, allergen, or heavy metal particles present in polluted city environment [28]. HPMC powder or HPMC in saline or salt water is already marketed in certain countries as a preventive film forming barrier against allergens but in the absence of any mechanism to stabilize the HPC / HPMC gel on the NM, its duration of action and in consequence its efficacy, cannot be guaranteed [21]. (Popov et al. 2020) demonstrated the in vitro efficacy of 5\% HPMC gel and in vivo safety in rats but the concentration of HPMC used for in vitro tests was too high as it forms a nearly solid gel through which any particulate matter cannot diffuse [21]. They also demonstrated that HPMC enhances decongestion through mucoadhesion, acts as a NM barrier in allergic rhinitis, without affecting efficacy of oxymetazoline for a week after its discontinuation (Valerieva et al. 2015) but AR preventing clinical efficacy of HPMC alone still remains to be validated [31].

The results of PCNS v/s HPMC film to prevent the diffusion of ragweed, 2 different dust mite allergens, alternaria-fungus allergen, and cat allergen, clearly demonstrate that PCNS forms a more impermeable allergen barrier compared to HPMC. These strong in vitro allergen preventing barrier properties of PCNS are probably related to the fact that glycerol and HPC molecules in PCNS form a mesh-like structure which cannot be penetrated by allergens, blocking the diffusion of allergens towards the agar gel. The second property of PCNS film is that the film contains osmotic glycerol while the underlying agar gel is isotonic. Being osmotic, the PCNS film may attract liquid from isotonic agar gel thereby inhibiting allergen diffusion towards the agar gel. Clinical results confirm 
these significant allergen and pollution barrier forming properties of PCNS film as all the AR clinical symptoms started reducing right after the first few days of application. Surprisingly, saline solution also showed considerable improvements in clinical AR and respiratory symptoms which may have been due to continuous cleaning of the NM, 3-4 times per day. Regular cleaning the NM surface would have minimized NM exposure to allergens and mechanical removal of inflammatory cytokines, providing possibilities of NM repair in a clean environment. PCNS acts identically to saline water induced mechanical cleaning of the NM but its additional osmotic activity and prolonged stability would have helped a rapid and efficient removal of allergens and surface contaminants. Glycerol being cell friendly and polymers being inert, the mode of action of PCNS film remains mechanical without any cellular interactions without any serious side-effects.

Clinical observations reveal that although PCNS was applied only on the $\mathrm{NM}$, the ocular symptoms of allergy were also concomitantly reduced with the nasal symptoms. This is probably due to barrier forming properties of PCNS which minimizes allergen contact with the NM, suppresses allergen cascade, and decreases new allergen contact with circulating mast cells, leading to lower release of histamine and other proinflammatory proteins on the ocular surface.

The AR symptomatic relief with PCNS was much superior to controls (statistically significant from day-6 onwards for all the parameters, $\mathrm{p}<0.001)$ with continuous improvement during the entire 3-week treatment period. As these beneficial effects were progressive, they are considered to be related to preventive properties of the nasal filmogen barrier which reduces allergen contact with the nasal mucosa, thereby allowing reconstitution of the nasal mucosa and in consequence, better nasal mucosa defence as well as minimum pollution inflicted systemic consequences.

AR nasal symptoms, airflow and markers of inflammation directly affect the lower respiratory tract and correlate with the clinical expression of the allergic airway syndrome $[11,20]$. The incidence of allergic airway syndrome is rising continuously without any specific explanation and seems not to correlate with the decrease in infection with pathogenic organisms, wearing masks, getting vaccinated, and following good domestic hygiene [6]. We are getting more and more sensitive to infection and allergens and consequently we are using more and more chemical drugs to try to get symptomatic relief. Continuous and chronic use of chemical drugs to treat AR, hygiene, and cleanliness is most likely responsible of suppressing our immune system and make us prone to allergens. We may not get an immediate reply, but we need to find effective, multi-target, non-chemical, and safe solutions to tackle epidemics of allergic airway syndrome. This was not possible because we could not find multi-target drugs but a simple NM barrier which can stop or at least minimize contact of allergenic particles with the NM, along with minimizing inflammation and allowing NM mucosa repair, without any side effects, can provide an excellent alternative.

Being a liquid mask, PCNS is now registered in Europe as a medical device and the properties of the liquid bandage are now being adjusted to treat other topical infections and injuries.

Conclusion: Applying a polymeric, osmotic, stable, and nonchemical film on the nasal surface instead of continuously using chemical drugs, represents a safe approach in preventing pollution and allergen induced respiratory symptoms.

Abbreviations: HPMC (hydroxypropyl methylcellulose), NM (Nasal Mucosa), AR (Allergic Rhinitis), ELISA (Enzyme-Linked ImmunoSorbent Assay), As (Arsenice), $\mathrm{Pb}$ (Lead), Cd (Cadmium), $\mathrm{Hg}$ (Mercury)

\section{References}

1. Ahmed, Tarek A., Khalid M. El-Say, Osama A. A. Ahmed, and Ahmed S. Zidan. (2018). 'Chapter 9 - Sterile Dosage Forms Loaded Nanosystems for Parenteral, Nasal, Pulmonary and Ocular Administration'. In Nanoscale Fabrication, Optimization, ScaleUp and Biological Aspects of Pharmaceutical Nanotechnology, ed. Alexandru Mihai Grumezescu. William Andrew Publishing, 335-395. (November 22, 2021).

2. Anto, Josep M. et al. (2017). 'Mechanisms of the Development of Allergy (MeDALL): Introducing Novel Concepts in Allergy Phenotypes'. Journal of Allergy and Clinical Immunology 139(2): 388-399.

3. 'Asthma'. (November 22, 2021).

4. Bédard, A. et al. (2020). 'Treatment of Allergic Rhinitis during and Outside the Pollen Season Using Mobile Technology. A MASK Study'. Clinical and Translational Allergy. 10(1):62.

5. Bjermer, Leif, Marit Westman, Mats Holmström, and Magnus C. Wickman. (2019). 'The Complex Pathophysiology of Allergic Rhinitis: Scientific Rationale for the Development of an Alternative Treatment Option'. Allergy, Asthma, and Clinical Immunology: Official Journal of the Canadian Society of Allergy and Clinical Immunology 15: 24.

6. Bloomfield, SF, R Stanwell-Smith, RWR Crevel, and J Pickup. (2006). 'Too Clean, or Not Too Clean: The Hygiene Hypothesis and Home Hygiene'. Clinical and Experimental Allergy 36(4): 402-425.

7. Bousquet, Jean et al. (2020). 'Allergic Rhinitis'. Nature Reviews Disease Primers 6(1): 1-17.

8. Bousquet, P.-J. et al. (2008). 'Geographical Distribution of Atopic Rhinitis in the European Community Respiratory Health Survey I'. Allergy 63(10): 1301-1309.

9. Braunstahl, G.-J. et al. (2003). 'Mucosal and Systemic Inflammatory Changes in Allergic Rhinitis and Asthma: A Comparison between Upper and Lower Airways'. Clinical and Experimental Allergy: Journal of the British Society for Allergy and Clinical Immunology 33(5): 579-587.

10. Cayrol, Corinne et al. (2018). 'Environmental Allergens Induce Allergic Inflammation through Proteolytic Maturation of IL-33'. Nature Immunology 19(4):375-385.

11. Compalati, Enrico et al. (2010). 'The Link between Allergic Rhinitis and Asthma: The United Airways Disease'. Expert Review of Clinical Immunology 6(3):413-423.

12. 'Biological Evaluation of Medical Devices - Part 5: Tests Concerning In Vitro Cytotoxicity". In NF EN ISO 10993-10995.

13. Eyerich, Stefanie, Martin Metz, Apostolos Bossios, and Kilian Eyerich. (2020). 'New Biological Treatments for Asthma and Skin Allergies'. Allergy 75(3): 546-560.

14. Ferreira, Manuel A et al. (2017). 'Shared Genetic Origin of Asthma, Hay Fever and Eczema Elucidates Allergic Disease Biology'. Nature genetics 49(12): 1752-1757.

15. Juniper, E. F., and G. H. Guyatt. (1991). 'Development and Testing of a New Measure of Health Status for Clinical Trials in Rhinoconjunctivitis'. Clinical and Experimental Allergy: Journal of the British Society for Allergy and Clinical Immunology 21(1): 77-83.

16. Kim, Dasom, Zi Chen, Lin-Fu Zhou, and Shou-Xiong Huang. (2018). 'Air Pollutants and Early Origins of Respiratory Diseases'. Chronic Diseases and Translational Medicine 4(2): 7594.

17. Kim, Jihyun et al. (2020). 'The Effect of Air Pollutants on Airway Innate Immune Cells in Patients with Asthma'. Allergy 75(9): 2372-2376.

18. Lambrecht, Bart N., and Hamida Hammad. (2014). 'Allergens and the Airway Epithelium Response: Gateway to Allergic 
Sensitization'. Journal of Allergy and Clinical Immunology 134(3): 499-507.

19. Malone, Michael. 'Review: Side Effects of Some Commonly Used Allergy Medications (Decongestants, Anti-Leukotriene Agents, Antihistamines, Steroids, and Zinc) and Their Safety in Pregnancy'. (November 22, 2021).

20. de Oliveira, Tiago Bittencourt, Everton Andrei Klering, and Ana Beatriz Gorini da Veiga. (2019). 'Is Recurrent Respiratory Infection Associated with Allergic Respiratory Disease?' The Journal of Asthma: Official Journal of the Association for the Care of Asthma 56(2): 160-166.

21. Popov, Todor A., Jean Emberlin, Peter Josling, and Alexander Seifalian. (2020). 'In Vitro and in Vivo Evaluation of the Efficacy and Safety of Powder Hydroxypropylmethylcellulose as Nasal Mucosal Barrier'. Medical Devices (Auckland, N.Z.) 13:107-113.

22. Roan, Florence, Kazushige Obata-Ninomiya, and Steven F. Ziegler. (2019). 'Epithelial Cell-Derived Cytokines: More than Just Signaling the Alarm'. The Journal of Clinical Investigation. 129(4):1441-1451.

23. Schrage, Arnhild et al. (2011). 'Bovine Corneal Opacity and Permeability Test in Routine Ocular Irritation Testing and Its Improvement within the Limits of OECD Test Guideline 437'. Alternatives to laboratory animals. (October 14, 2020).

24. Shamji, Mohamed H. et al. 'The Role of Allergen-Specific IgE, IgG and IgA in Allergic Disease'. Allergy n/a(n/a). (November 22, 2021).

25. Shrivastava, Lea, Hendrik Schütte, Pawan Malik, and Ravi Shrivastava. (2017). 'A New Class of Polymeric Anti-Allergen Nasal Barrier Film Solution for the Treatment of Allergic Rhinitis'. Journal of Allergy \& Therapy. 08(03). (November 16, 2021).

26. Shrivastava, Ravi, Remi Shrivastava, Bianca Johansen, and Thibault Allain. (2021). 'Anti-Inflammatory and Antiviral
Osmotic Polymeric Film to Treat Covid-19 Early-Stage Infection'. Journal of Inflammation Research. 14:1195-1206.

27. Shrivastava, Remi, Lea Shrivastava, and Ravi Shrivastava. (2016). 'Composition for Topical Application Comprising Glycerol and Tannins'. (November 16, 2021).

28. Shrivastava, Remi, Megha Vijay, Nathalie Maneby, and Ravishekhar Shrivastava. (2021). " $<\mathrm{p}>$ Clinical Efficacy of an Osmotic, Antiviral and Anti-Inflammatory Polymeric Nasal Film to Treat Covid-19 Early-Phase Respiratory Symptoms $</ \mathrm{P}>$ '. Open Access Journal of Clinical Trials. 13:11-20.

29. Subbarao, Padmaja et al. (2015). 'The Canadian Healthy Infant Longitudinal Development (CHILD) Study: Examining Developmental Origins of Allergy and Asthma'. Thorax. 70(10):998-1000.

30. Tran, Thach Quang et al. (2021). 'Efficacy of Face Masks against Respiratory Infectious Diseases: A Systematic Review and Network Analysis of Randomized-Controlled Trials.' Journal of breath research (Online). (November 22, 2021).

31. Valerieva, Anna et al. (2015). 'Effect of Micronized Cellulose Powder on the Efficacy of Topical Oxymetazoline in Allergic Rhinitis'. Allergy and Asthma Proceedings. 36(6):134-139.

32. Vieira, Melissa Gurgel Adeodato, Mariana Altenhofen da Silva, Lucielen Oliveira dos Santos, and Marisa Masumi Beppu. (2011). 'Natural-Based Plasticizers and Biopolymer Films: A Review'. European Polymer Journal. 47(3):254-263.

33. Watts, Annabelle M., Allan W. Cripps, Nicholas P. West, and Amanda J. Cox. (2019). 'Modulation of Allergic Inflammation in the Nasal Mucosa of Allergic Rhinitis Sufferers with Topical Pharmaceutical Agents'. Frontiers in Pharmacology 10: 294.

34. Wheatley, Lisa M., and Alkis Togias. (2015). 'Allergic Rhinitis'. (November 16, 2021).

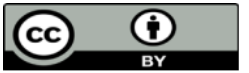

This work is licensed under Creative Commons Attribution 4.0 License

To Submit Your Article Click Here: Submit Manuscript

DOI: $10.31579 / 2690-1919 / 219$
Ready to submit your research? Choose Auctores and benefit from:

$>$ fast, convenient online submission

$>$ rigorous peer review by experienced research in your field

$>$ rapid publication on acceptance

$>$ authors retain copyrights

$>$ unique DOI for all articles

$>$ immediate, unrestricted online access

At Auctores, research is always in progress.

Learn more https://auctoresonline.org/journals/journal-of-clinical-researchand-reports- 